\title{
Buoyancy increase and drag-reduction through a simple superhydrophobic coating
}

Gi Byoung Hwang a, Adnan Patir ${ }^{\text {a }}$, Kristopher Page ${ }^{\text {a }}$, Yao Lu ${ }^{\text {a }}$, Elaine Allan ${ }^{\mathrm{b}}$ and Ivan P. Parkin ${ }^{a *}$

${ }^{a}$ Materials Chemistry Research Centre, Department of Chemistry, University College

London, 20 Gordon Street, London, WC1H OAJ, United Kingdom

${ }^{b}$ Division of Microbial Diseases, UCL Eastman Dental Institute, University College London, 256 Gray's Inn Road, London WC1X 8LD, United Kingdom

* To whom correspondence should be addressed.

E-mail: i.p.parkin@ucl.ac.uk Tel: 44(0)207 6794669 


\begin{abstract}
A superhydrophobic paint was fabricated using $1 \mathrm{H}, 1 \mathrm{H}, 2 \mathrm{H}, 2 \mathrm{H}$-perfluorooctyltriethoxysilane (PFOTES), $\mathrm{TiO}_{2}$ nanoparticles and ethanol. The paint has potential for an aquatic application of a superhydrophobic coating as it induced increased buoyancy and drag reduction. Buoyance testing showed that the reduction of surface energy by superhydrophobic coating made it feasible that glass, a high density material, was supported by the surface tension of water. In a miniature boat sailing test, it was shown that the low energy surface treatment decreased the adhesion of water molecules to the surface of the boat resulting in a reduction of the drag force. Additionally, a robust superhydrophobic surface was fabricated through layer-by-layer coating using adhesive tape and the paint, after $100 \mathrm{~cm}$ abrasion test with sand paper, the surface still kept its water repellency, enhanced buoyancy and drag reduction.
\end{abstract}




\section{Introduction}

Controlling wettability of solid surfaces has been an important issue attracting increasing interest from fundamental and practical perspectives ${ }^{1}$. The surface wettability is mostly measured by means of water contact angle on a solid surface. When the water contact angle is $>150^{\circ}$, the surface becomes superhydrophobic ${ }^{2}$. Superhydrophobic surfaces, are extremely water repellent, they make water droplets roll and enable the droplet to carry away dirt without wetting ${ }^{3}$. Many techniques to fabricate superhydrophobic surfaces on substrates have been proposed by combining suitable surface roughness along with low surface energy materials, such methods include chemical vapor deposition, plasma etching, polymerization, and sol-gel process, and the drawback of superhydrophobic coating is that they are mechanically weak ${ }^{4-12}$.

In nature, the water strider is an insect that uses superhydrophobic surfaces ${ }^{13}$. The hierarchical structures (nano-sized hairs with nano grooves) of the strider's leg allow the insect to float, slide, and jump on the surface of water, and a single leg of the insect can support approximately 15 times its total body weight ${ }^{13-15}$. This shows that aquatic application of superhydrophobic surfaces has the potential to significantly improve buoyancy and/or reduce water drag. Mimicking the structure of the strider's leg has attracted significant attention for formation of bioinspired devices. Although legs made of superhydrophobic wires allow an artificial small insect shaped craft to float on water, the supporting force is relatively small ${ }^{14,16}$. Previous studies have shown that a superhydrophobic coating made it possible for a box made with copper mesh to float on water, the coating that was applied to the miniature boat resulted in an increased loading capacity of several hundred milligrams, 
and the phenomenon was believed to have arisen from the air bubbles entrapped by the superhydrophobic surface ${ }^{17}{ }^{18}$

With respect to drag resistence, it is currently under debate whether a superhydrophobic coating can decrease drag reduction. A numerical study reported that the surface roughness produced water drag reduction and that its effectiveness is higher at turbulence ${ }^{19}$. However, Steinberger's group studied superhydrophobic surfaces embedded within a square lattice of calibrated cylindrical holes and they showed that gas entrapped at the surface acted as an anti-lubricant and produced high water friction ${ }^{20} . \mathrm{Su}$ and co-workers reported that a superhydrophic coating on spheres increased water drag when submerged whereas it reduced the drag for the motion on water, and it was speculated that the plastron property of bubble layer trapped at the superhydrophobic surface played an important role in altering drag coefficient $^{21}$.

In this study, we report a superhydrophobic paint which can be readily applied to various surfaces through dipping, painting, and spraying. Our superhydrophobic coating on glass, which is a high density material, enable it float on water, even after significant weight loading. A boat sailing test showed that the superhydrophobic coating reduced the water drag force. It was concluded that the mechanism of the change of buoyancy and drag is largely attributed to surface energy reduction resulting from the superhydrophobic coating. 


\section{Results and Discussion}

$1 \mathrm{H}, 1 \mathrm{H}, 2 \mathrm{H}, 2 \mathrm{H}$-perfluorooctyltriethoxysilane (PFOTES), $\mathrm{TiO}_{2}$ nanoparticles and, ethanol were employed in order to make a superhydrophobic paint. As shown in Figure $1, \mathrm{TiO}_{2}$ nanoparticles were mixed with ethanol solution containing PFOTES and then vortexed for 5 min. In this process, PFOTES, forms a self-assembling monolayer, covalently attached to the surface of $\mathrm{TiO}_{2}$ nanoparticles ${ }^{22,} 23$. Figure $\mathrm{S} 1$ shows that after PFOTES treatment, $\mathrm{TiO}_{2}$ nanoparticles changed from hydrophilicity to hydrophobicity.

To characterise the paint, glass slides were coated and then dried for $3 \mathrm{~h}$ in the dark. Scanning electron microscopy (SEM) was used to examine the topography of painted surfaces and coating thickness. As shown Figure 2 (a), SEM analysis showed that after drying, fine cracks with a thickness of $\sim 5 \mu \mathrm{m}$ were formed across the painted surface and the agglomerated $\mathrm{TiO}_{2}$ nanoparticles produced a rough surface texture. Additionally, the coating thickness of the paint on the glass was $\sim 171 \mu \mathrm{m}$. X-ray photoelectron spectroscopy measurement was performed to investigate the surface chemistry of the treated surface. As shown Figure 2 (b), $\mathrm{Ti}$ and $\mathrm{O}$ signals are for $\mathrm{TiO}_{2}$, and other signals in the spectra are attributed to the PFOTES molecules. Specially Si and F peaks are obvious and provide direct evidence of the PFOTES existence on the surface of $\mathrm{TiO}_{2}$. Fluorine distribution across the surface significantly reduces the surface energy of the treated surface, and the rough surface structure with low surface energy led to water repellency.

The painted surface gave water contact angles of $>160^{\circ}$, and contact angle hysteresis and rolling off angle of $<5^{\circ}$, indicating superhydrophobicity. A water dropping test on the painted surface showed that water droplets bounced and rolled off the surface without wetting 
(Figure 3(a)). The self-cleaning test showed that after dropping water, dirt was carried away as the droplets rolled off the surface of the painted glass (Figure 3(b)).

To determine the buoyancy boost induced by the superhydrophobic paint coating, glass slides were used. It is known that glass submerges in water because it has higher density (2.4 $\left.\mathrm{g} / \mathrm{cm}^{3}\right)$ than water $\left(1.0 \mathrm{~g} / \mathrm{cm}^{3}\right)$, and it is often readily wettable ${ }^{24,25}$. However, our experiments showed that despite the material having significantly higher density than water, superhydrophobic coated glass slides can float on water. As shown in Figure 4 (a), uncoated glass slide sank in water right after it was put on the water surface. However, interestingly, after application of the superhydrophobic coating, the samples (edge, top, and whole surface coated samples) stayed on the water, and water dimples were observed around the samples (Figure 4(a)). Moreover, the interface between the glass and water were different depending on the position of the painted surface (Figure S2). A glass slide coated on bottom with a superhydrophobic paint showed that although the painted surfaces have a significant amount of air entrapped between them and the water, the buoyancy offered by this thin air layer was not sufficient for the glass to float on water (Figure 4(a) and Figure S3). The painted glass samples are mainly supported by the surface tension of water. The surface tension is determined by the surface energy of the material with higher surface energy, indicating greater molecular attraction ${ }^{26,27}$. The surface energy of glass (soda-lime glass: $83.4 \mathrm{~mJ} / \mathrm{m}^{2}$ ) is higher than that of water (surface energy: $72 \mathrm{~mJ} / \mathrm{m}^{2}$, equivalent to surface tension), indicating that glass tends to attracts water ${ }^{28-30}$. However, the superhydrophobic paint coating ensure that the glass samples has a lower surface energy (surface energy: $<6.7 \mathrm{~mJ} / \mathrm{m}^{2}$ ) than that of water ${ }^{28,29}$, and this results in reduced interaction between the glass and the water (Figure S4).

In weight loading tests of the edge-, top-, and whole surface-coated samples, Figure 4 (b), the loading capacity of the treated glass samples was proportional to the position of coating on the substrate (all samples: P-value <0.05). The edge-coated samples with mass $4.7 \mathrm{~g}$ (glass 
weight) carried a weight of $3.2 \mathrm{~g}\left(160 \mathrm{mg} / \mathrm{cm}^{2}\right)$ and produced a dimple of $3.5 \mathrm{~mm}$ depth without sinking (Figure 4(b), and Table S1). However, at weight loading of $>3.2 \mathrm{~g}$, the edgecoated sample sank in water because the untreated top surface became exposed to water (Figure S4). Preventing top surface wetting by superhydrophobic coating induced higher loading capacity than edge-coated sample; top-coated sample carried a weight load of $4 \mathrm{~g}$ $\left(200 \mathrm{mg} / \mathrm{cm}^{2}\right)$. The glass coated on all sides carried a weight loading of $6.3 \mathrm{~g}\left(319 \mathrm{mg} / \mathrm{cm}^{2}\right)$ and produced a dimple of $4.8 \mathrm{~mm}$ depth (Figure 4 (b), and Table S1). This was $58 \%$ increase of loading capacity, compared to top-coated samples. We attribute this to water surface tension and a significant amount of air bubbles entrapped at the treated surface subsequently increased buoyancy (Figure S5).

To determine the reduction in water drag force reduction by the superhydrophobic coating, a sailing test of untreated and treated boats was conducted. As shown in Figure S5, the boat was filled with $100 \mathrm{~g}$ of glass beads, this then was placed on the surface of the water. The boat was connected to weights via a string. The pulling force of the weight under gravity allowed the boat to move along straight path on the water. Figure 5 (a) shows a sailing test of the boat before and after superhydrophobic coating. Each experiment was repeated 6 times. Under applied force ranging from 17.6 to $47 \mathrm{mN}$, the sailing velocities of boat with superhydrophobic coating were always higher than the velocities of untreated boat. (P-value $<0.01)$. A comparison of the velocities of the two boats showed that the superhydrophobic coating reduced the water drag force by maximum $12.7 \%$ at $47 \mathrm{mN}$.

Figure 5 (b) shows that water adhesion forces on the boat were reduced by the superhydrophobic coating. The shape of the water surface close to the surface of the untreated boat was a concave meniscus, indicating that the adhesion forces between water molecules and the boat are greater than the cohesion forces between the water molecules ${ }^{31}$. After superhydrophobic coating, the water surface shape near to the boat changed to a convex 
meniscus, indicating that the adhesion forces of the water molecules on the surface of the boat decreased ${ }^{31}$.

Previous studies speculated that microbubbles trapped at the solid/water interface played an important role in the water drag reduction ${ }^{21,32,33}$. However, Steinerger's group demonstrated that the menisci of microbubbles has a dramatic influence on the boundary condition of the water and the surface and can turn it from slippery to sticky ${ }^{20}$. Additionally, Shi's group showed that the water capture of superhydrophobic surface can determine the drag-reducing (no water captured) or drag-increasing (water captured) ${ }^{34}$. Most studies have focused on surface structure or air bubbles on the surface to explain the water drag of superhydrophobic surface whereas they have not considered the reduction effect of surface energy. It is well known that the viscosity of a liquid is an important factor affecting the friction between water and a solid surface, indicating that a less adhesive liquid produces less friction ${ }^{35}$. Our experiment showed that the surface energy reduction by a superhydrophobic coating decreased the adhesion force of water molecules on the boat leading to a water drag reduction for the sailing boat.

Maritime shipping is the most carbon efficient form of transportation in the world, and it accounts for $2-15 \%$ of global gas $\left(\mathrm{SO}_{\mathrm{x}}, \mathrm{CO}, \mathrm{CO}_{2}\right.$, and $\left.\mathrm{NO}_{\mathrm{x}}\right)$ emission, affecting global warming 36,37 . The reduction in water drag force, an undesirable phenomenon, is one of the most significant challenges in the shipping industry because it would benefit the global environment and would also provide energy and cost savings. Our experimental results show that a superhydrophobic coating is an effective means to reduce the water drag force of a miniature boat.

For its aquatic application in the real world, it is necessary for the surface to be durable because water-friction and undesirable aquatic residues or debris may damage the surface. Here, a robust superhydrophobic surface was produced through layer-by-layer coating using 
adhesive tape and the paints and the mechanical durability of the surface was tested under extreme environments. As shown Figure S5, the treated sample was pressed by a $100 \mathrm{~g}$ weight and made to slide on sand paper at constant speed. The water repellency of the surface during abrasion test was investigated. As shown in Figure 6 (a), after $100 \mathrm{~cm}$ of sliding friction, the treated sample retained its surperhydrophobicity with water contact angles of $>158^{\circ}$ and contact angle hysteresis of $<5^{\circ}$ and, a minor increase of the rolling off angle was observed from $0^{\circ}$ to $3^{\circ}$. Even after the abrasion, the treated samples still caused the enhanced buoyancy and water drag reduction (Figure $6(\mathrm{~b}))$.

\section{Conclusion}

In summary, superhydrophobic paint was fabricated and its potential for aquatic application was determined. Buoyancy testing showed that the reduction of surface energy by the superhydrophobic coating makes it feasible that glass is supported by surface tension of water, and that the sailing test showed that the low surface energy decreased adhesion of water molecules on the surface of boat, resulting in a reduction of the water drag force. Additionally, on curved vehicles with large surface area, conventional superhyrophobic coating methods such as chemical vapor deposition, plasma etching, and sol-gel process are hard to apply for real world application. However, the superhydrophobic paint which we have developed can be readily applied to surfaces via painting and spraying regardless of the size and structure of the aquatic vehicle and robust superhydrohpobic surface can be easily fabricated as the paint is incorporated with the adhesive. 


\section{Experimental section}

\subsection{Preparation and sample coating of superhydrophobic paint}

$1.0 \mathrm{~g}$ of $1 \mathrm{H}, 1 \mathrm{H}, 2 \mathrm{H}, 2 \mathrm{H}$-perfluorooctyltriethoxysilane (PFOTES, $\mathrm{C}_{8} \mathrm{~F}_{13} \mathrm{H}_{4} \mathrm{Si}\left(\mathrm{OCH}_{2} \mathrm{CH}_{3}\right)_{3}$, Sigma-Aldrich, St. Louis, MO, USA) was mixed with $99.0 \mathrm{~g}$ of pure ethanol (EDM Millipore Co., Billerica, MA, USA), and it was stirred for 5 min. $13 \mathrm{~g}$ of Degussa P $25 \mathrm{TiO}_{2}$ nanoparticles were added into the solution with constant stirring, and the solution was vortexed for $5 \mathrm{~min}$.

\subsection{Water repellent test and water contact angle}

To determine water repellency, soda-lime glass slides (size: $2.6 \times 7.6 \mathrm{~cm}$, VWR international, Radnor, PA, USA) were coated using $450 \mu \mathrm{L}$ of the paint, and air-dried in the dark for 3 h. $10 \mathrm{~mL}$ of water mixed with Congo red dye (Sigma-Aldrich, St. Louis, MO, USA) was dropped onto the tilted painted surface using a pipette.

The water contact angle on the painted surface was measured using a contact angle meter (First Ten Angstroms, Inc., Portsmouth, Virginia, USA). A droplet $(\sim 5 \mu \mathrm{L})$ of DI water was put onto the surface, photographed side on and the images were analyzed using Surftens 4.5 software. The contact angle hysteresis $(\mathrm{CAH})$ on the painted surface was investigated by "add and remove volume" method. The CAH was determined by the difference between advanced angle and receding angle ${ }^{38}$.

\subsection{SEM Analysise}

To investigate the surface morphology and coating thickness, the samples were coated by a fine layer of gold to inhibit charging. Scanning Electron Microscopy (SEM, JEOL Inc., Peabody, MA, USA) was used at an accelerating voltage of $5 \mathrm{kV}$. Images were captured using SEMAfore software. 


\subsection{Buoyancy and loading capacity tests}

Three different samples were prepared; edge-, top-, bottom-, and whole surface-coated samples. The untreated sample and treated glass samples were put on the surface of water to determine if the samples stay on water surface. To investigate load capacity of the treated samples on water surface, $0.6 \mathrm{~g}$ or $0.3 \mathrm{~g}$ weights were used. The weights were gently loaded on the middle of the sample to maintain balance.

\subsection{Sailing test of boat}

A boat with superhydrophobic coating was prepared by dipping in the paint and then dried for $3 \mathrm{~h}$ in dark. To mimic boat containing freights, the boat (weight: $14 \mathrm{~g}$, volume; $8.5 \times 8.5 \times$ $5 \mathrm{~cm}$ ) was filled with $100 \mathrm{~g}$ of glass beads and it was placed on water in water tank with $120.1 \mathrm{~cm}$ length, and $60 \%(3.0 \mathrm{~cm})$ of the boat sit beneath water. The boat was connected with a weight through string. As the weight was dropped by gravity, it allowed the boat to sail straight way (Figure S6). The experiment was reproduced more than 10 times.

\subsection{Preparation and mechanical durability test of robust superhydrophobic surface}

The mechanical durability test was performed by sand paper abrasion. Adhesive double side tapes were attached on sample and then the paints were coated on the adhesive treated surface. After coating, the samples stay in dark for $6 \mathrm{~h}$, and then as shown in Figure S7, the painted sample was placed face down to sand paper (standard glass paper, grit no. 150). The treated sample was pressed on the sand paper by $100 \mathrm{~g}$ of weight and then the sample moved for $100 \mathrm{~cm}$ along ruler. The water contact angle and water rolling off angle of the painted surface were measured at intervals of $8 \mathrm{~cm}$ using contact angle meter. 


\section{References}

1. I. P. Parkin and R. G. Palgrave, J Mater Chem, 2005, 15, 1689.

2. M. Ma and R. M. Hill, Curr. Opin. Colloid Interface Sci., 2006, 11, 193-202.

3. Y. Lu, S. Sathasivam, J. Song, C. R. Crick, C. J. Carmalt and I. P. Parkin, Science, 2015, 347, 1132-1135.

4. D. K. Sarkar and N. Saleema, Surf. Coat. Technol., 2010, 204, 2483-2486.

5. H. Zhang, R. Lamb and J. Lewis, STAM, 2005, 6, 236-239.

6. H. Yang and P. Jiang, Langmuir, 2010, 26, 12598-12604.

7. R. Furstner, W. Barthlott, C. Neinhuis and P. Walzel, Langmuir, 2005, 21, 956-961.

8. Y. Wu, T. Hang, Z. Yu, L. Xu and M. Li, Chem. Commun., 2014, 50, 8405-8407.

9. X. Deng, L. Mammen, H. J. Butt and D. Vollmer, Science, 2012, 335, 67-70.

10. M. Cao, X. Song, J. Zhai, J. Wang and Y. Wang, J. Phys. Chem B, 2006, 110, 13072-13075.

11. W. Zhang, Z. Shi, F. Zhang, X. Liu, J. Jin and L. Jiang, Adv. Mater., 2013, 25, 2071-2076.

12. M. Cheng, G. Ju, C. Jiang, Y. Zhang and F. Shi, J. Mater. Chem. A, 2013, 1, 13411.

13. X. Gao and L. Jiang, Nature, 2004, 432, 36.

14. J. S. Koh, E. Yang, G. P. Jung, S. P. Jung, J. H. Son, S. I. Lee, P. G. Jablonski, R. J. Wood, H. Y. Kim and K. J. Cho, Science, 2015, 349, 517-521.

15. F. Shi, J. Niu, J. Liu, F. Liu, Z. Wang, X. Q. Feng and X. Zhang, Adv. Mater., 2007, 19, 22572261.

16. D. L. Hu, B. Chan and J. W. Bush, Nature, 2003, 424, 663-666.

17. Q. Pan and M. Wang, ACS Appl. Mater. Interfaces, 2009, 1, 420-423.

18. J. Guo, S. Yu, J. Li and Z. Guo, Chem. Commun., 2015, 51, 6493-6495.

19. H. Park, H. Park and J. Kim, Phys. Fluids, 2013, 25, 110815.

20. A. Steinberger, C. Cottin-Bizonne, P. Kleimann and E. Charlaix, Nat. Mater., 2007, 6, 665-668.

21. B. Su, M. Li and Q. Lu, Langmuir, 2010, 26, 6048-6052.

22. S. M. Barlow and R. Raval, Surf. Sci. Rep., 2003, 50, 201-341.

23. J. Cech and R. Taboryski, Appl. Surf. Sci., 2012, 259, 538-541

24. G. B. Hwang, E. Allan and I. P. Parkin, ACS Appl. Mater. Interfaces., 2015, DOI:

10.1021/acsami.5b06927.

25. F. M. White, Fluid mechanics, McGraw-Hill Education, London, 2011.

26. Fundamentals of Adhesion, http://www.duratech.com/wp-content/uploads/Fundamentals-

$$
\text { 27. Fundamentals of Adhesion }
$$

http://www.markingsystems.com/pdf/3mfundamentals of adhesion.pdf, 2016).

28. S. K. Rhee, J. Mater. Sci., 1977, 12, 823-824.

29. Surface energy, https://en.wikipedia.org/wiki/Surface energy, (accessed 10 May 2016).

30. S. K. Rhee, J. Mater. Sci., 1977, 12, 823-824.

31. T. Brown, H. E. Lemay, B. E. Burtsten, C. J. Murphy and P. M. Woodward, in Chemistry, Pearson, New Yorks, Twelfth edn., 2012, ch. 11, p. 438.

32. S. Zhang, X. Ouyang, J. Li, S. Gao, S. Han, L. Liu and H. Wei, Langmuir, 2015, 31, 587-593.

33. F. Shi, J. Niu, J. Liu, F. Liu, Z. Wang, X. Q. Feng and X. Zhang, Adv Mater, 2007, 19, 2257-2261.

34. M. Cheng, M. Song, H. Dong and F. Shi, Small, 2015, 11, 1665-1671.

35. P. S. Bernard, Fluid Dynamics, Cambridge University Press, 2015.

36. V. Eyring, J. Geophys. Res., 2005, 110.

37. J. J. Corbett, Science, 1997, 278, 823-824.

38. F.-M. Chang, S.-J. Hong, Y.-J. Sheng and H.-K. Tsao, Appl. Phys. Lett., 2009, 95, 064102. 


\section{Figure legends}

Figure 1. Preparation of superhydrophobic paint

Figure 2. (a) SEM image of glass slide coated by superhydrophobic paint, and (b) XPS pattern of superhydrophobic paint.

Figure 3. (a) Water repellent and (b) self-cleaning property of the coated glass slide

Figure 4. (a) Buoyancy test of control, bottom-, edge-, top-, and whole surface-coated glass microscope slides, and (b) loading capacity of edge-, top- and whole surface-coated samples.

Figure 5. (a) Sailing test of untreated boat and superhydrophobic paint coated boat, and (b) adhesion of water molecules on boat surface before and after superhydrophobic coating

Figure 6. (a) Estimation of water contact angle, contact angle hysteresis, and rolling off angle on treated samples after abrasion test using sand paper, and (b) treated glass sample floating on water and adhesion of water molecule on the surface of the treated boat after $100 \mathrm{~cm}$ abrasion test 

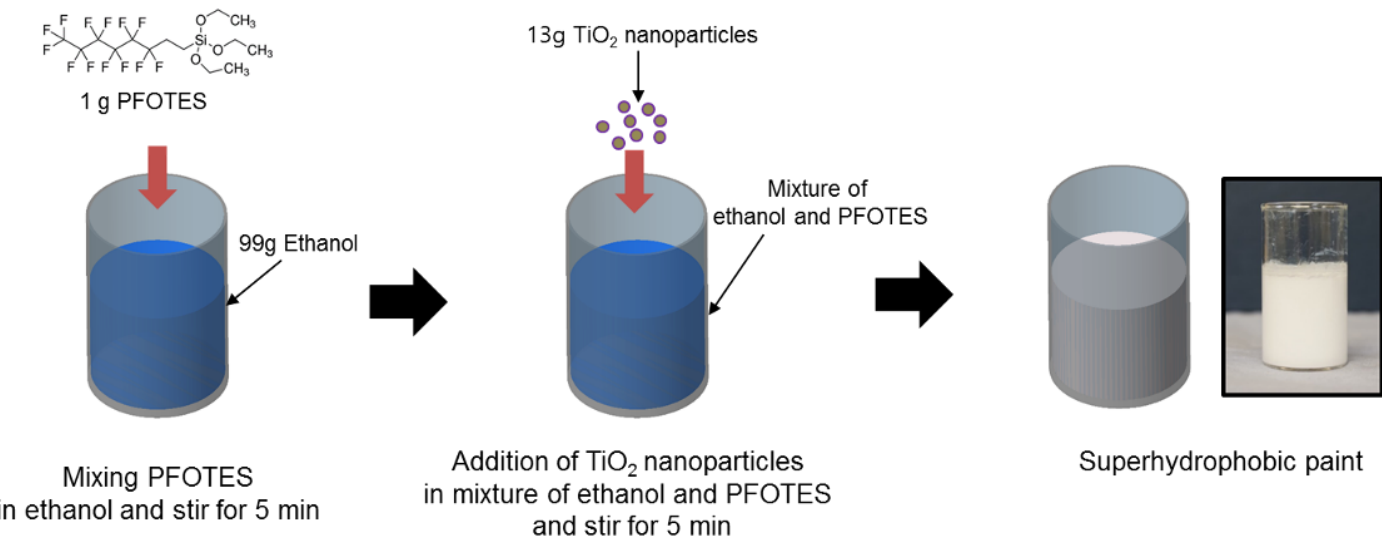

Superhydrophobic paint

Figure 1. Preparation of superhydrophobic paint 

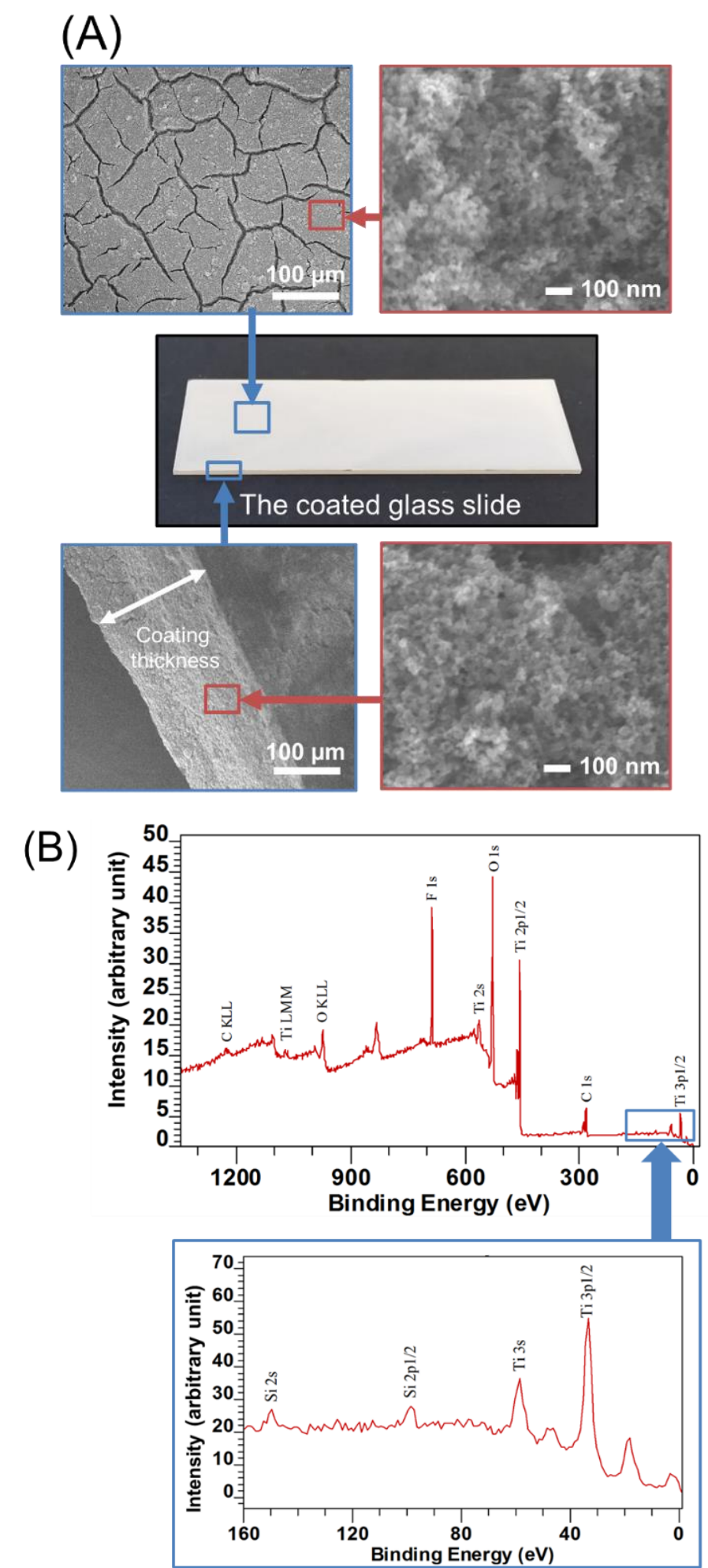

Figure 2. (a) SEM image of glass slide coated by superhydrophobic paint, and (b) XPS pattern of superhydrophobic paint. 

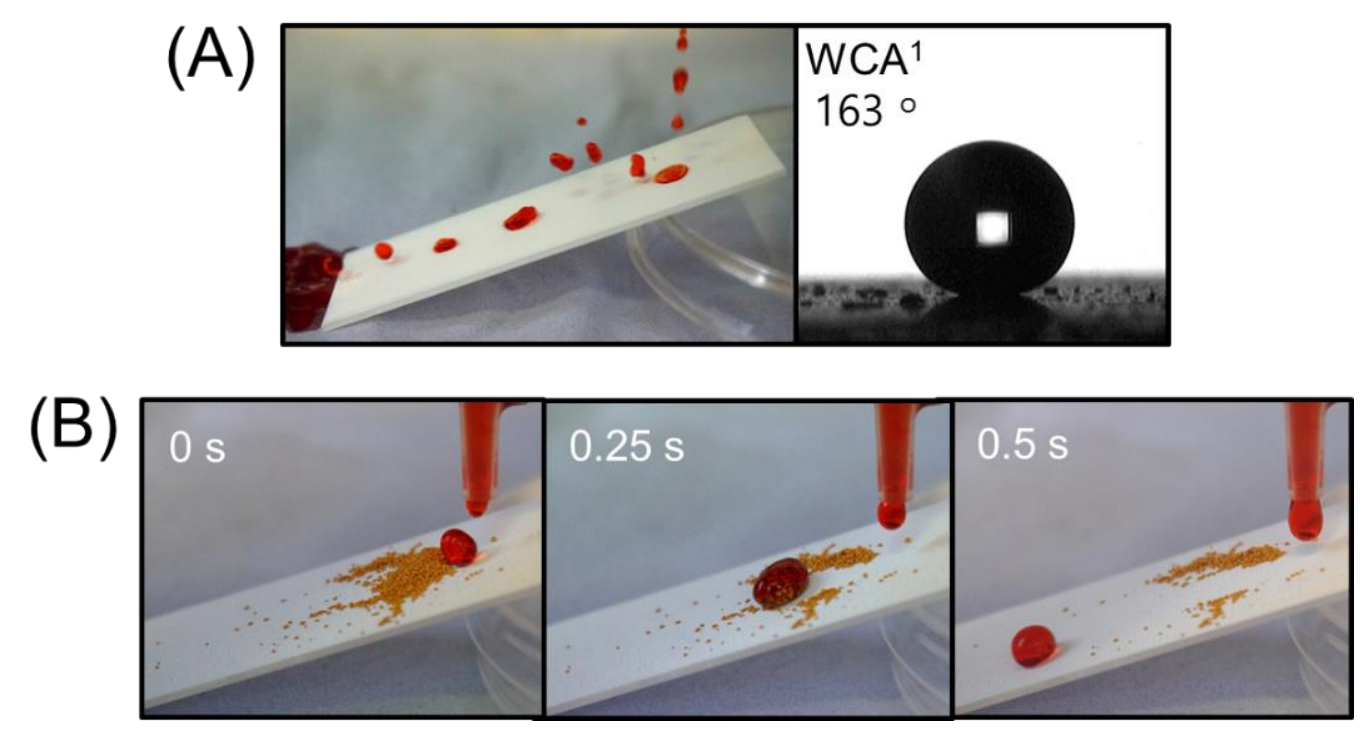

Figure 3. (a) Water repellent and (b) self-cleaning property of the coated glass slide ${ }^{1}$ WCA: water contact angle 


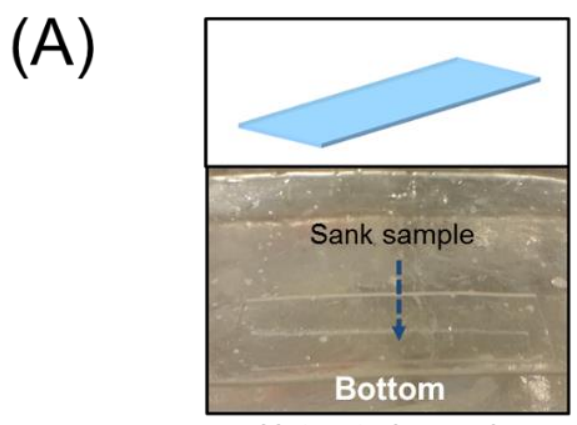

Untreated sample

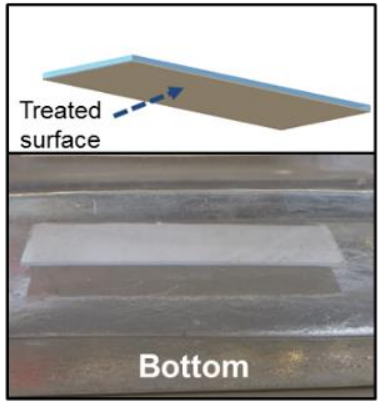

Bottom coated sample

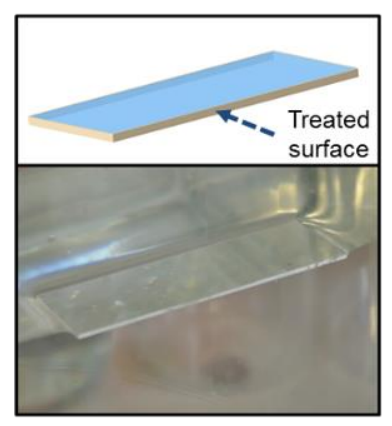

Edge coated sample

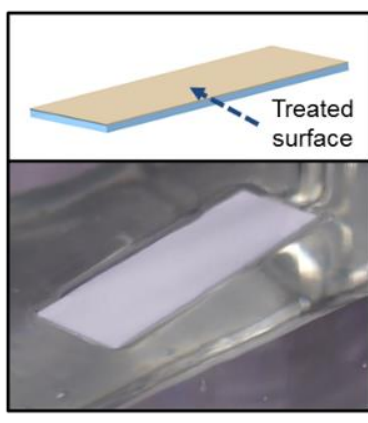

Top coated sample

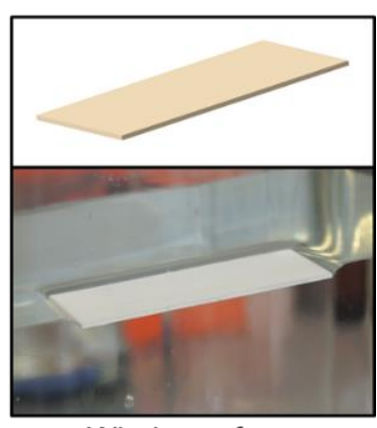

Whole surface coated sample

(B)

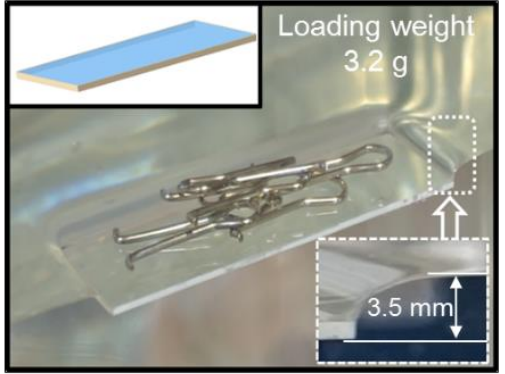

Edge coated sample

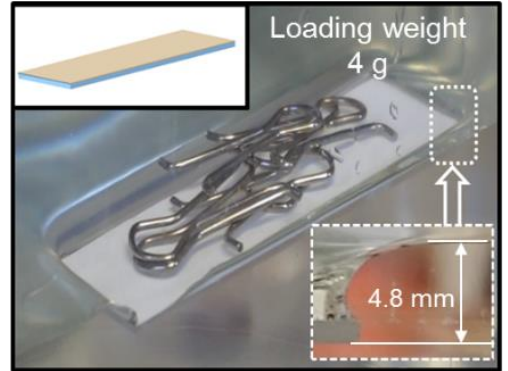

Top coated sample

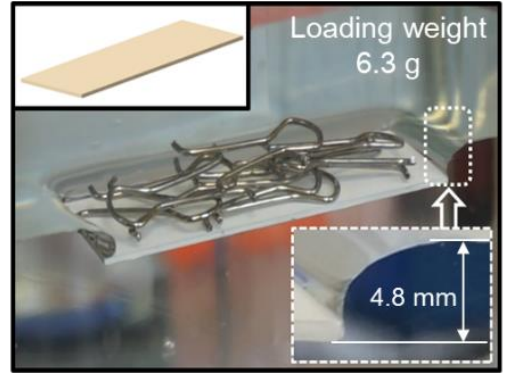

Whole surface coated sample

Figure 4. (a) Buoyancy test of control, bottom-, edge-, top-, and whole surface-coated glass microscope slides, and (b) loading capacity of edge-, top- and whole surface-coated samples. 


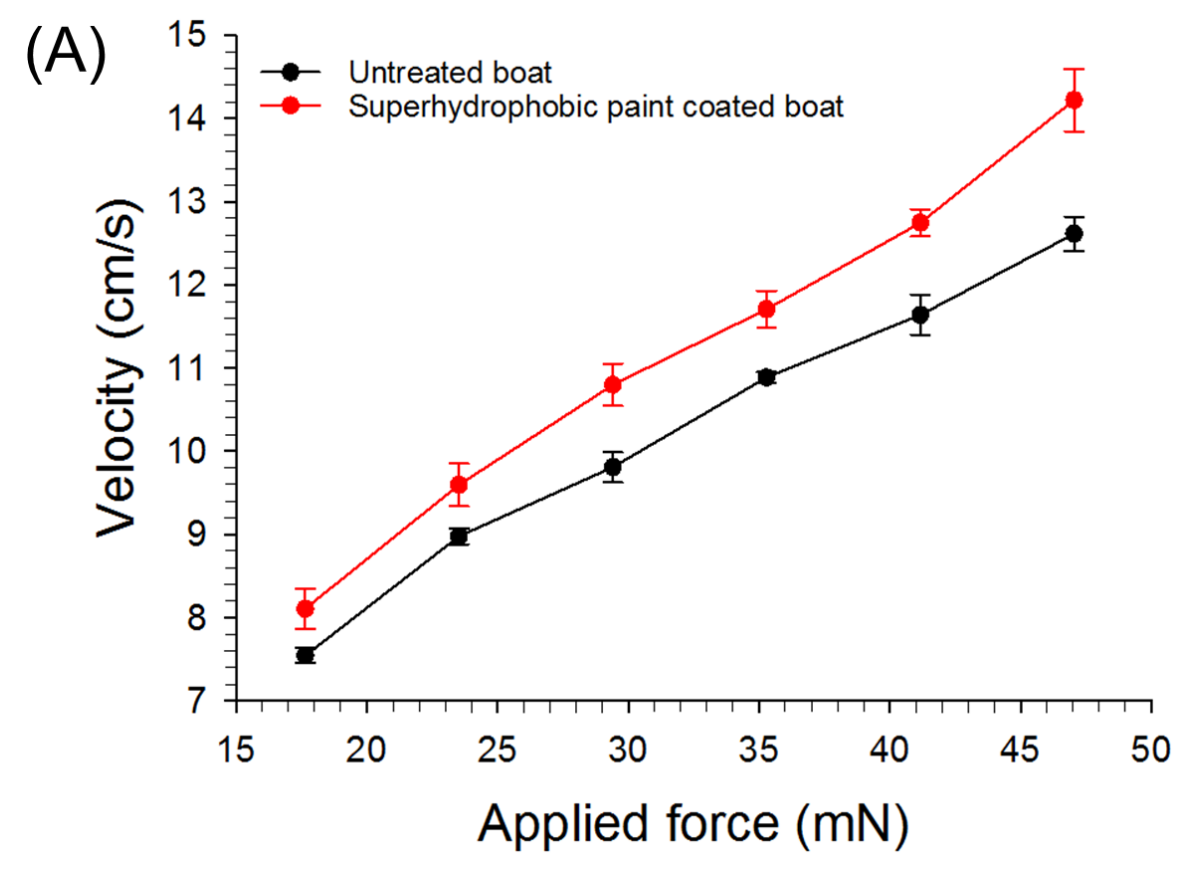

(B)
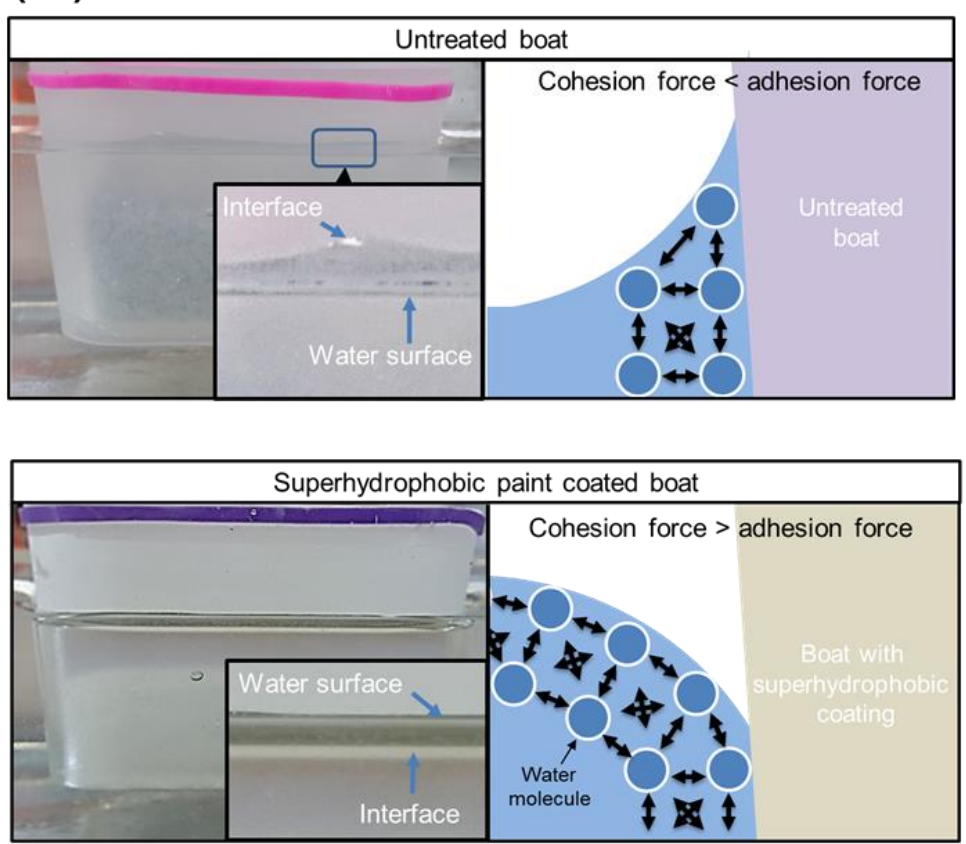

Figure 5. (a) Sailing test of untreated boat and superhydrophobic paint coated boat, and (b) adhesion of water molecules on boat surface before and after superhydrophobic coating 


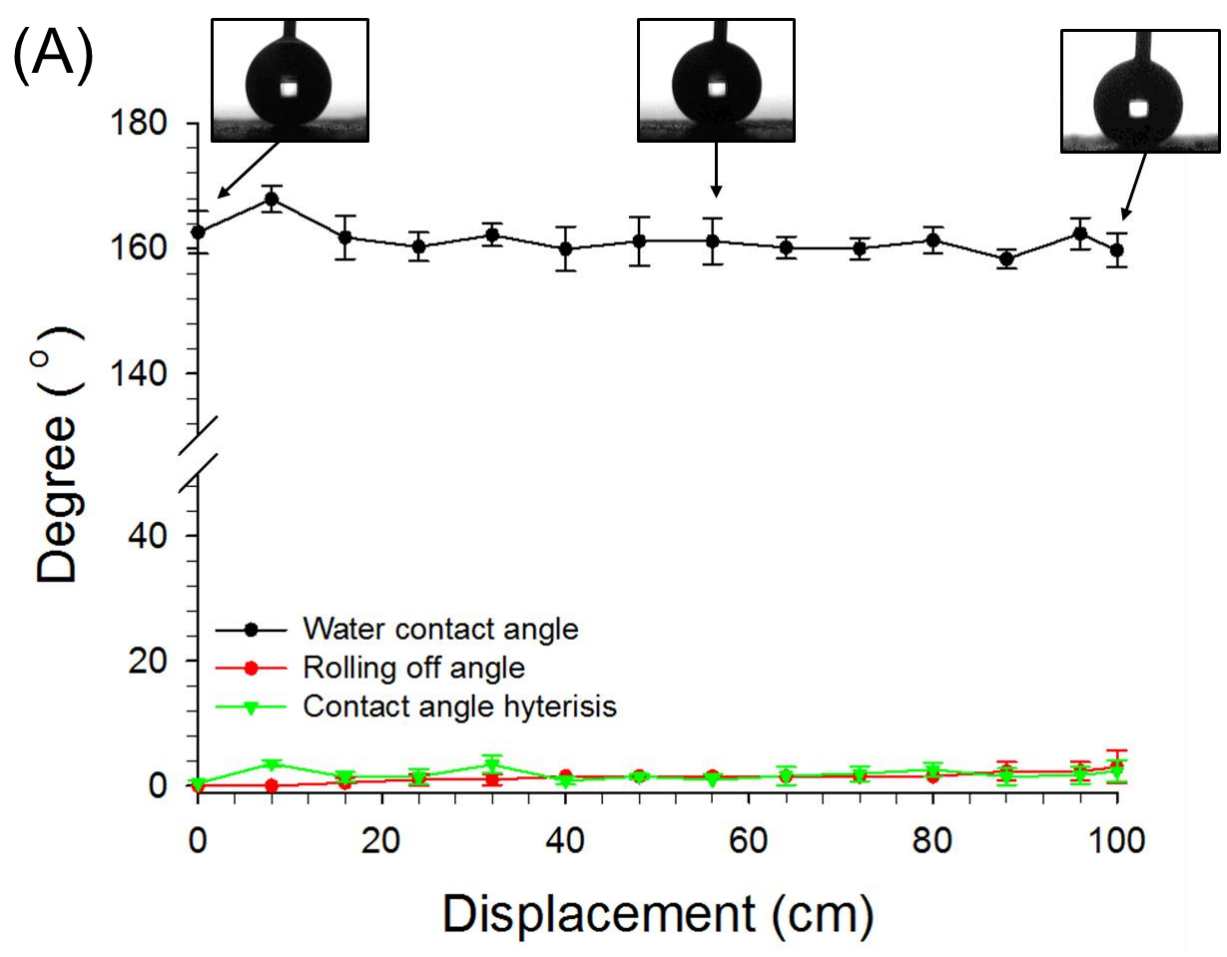

(B)

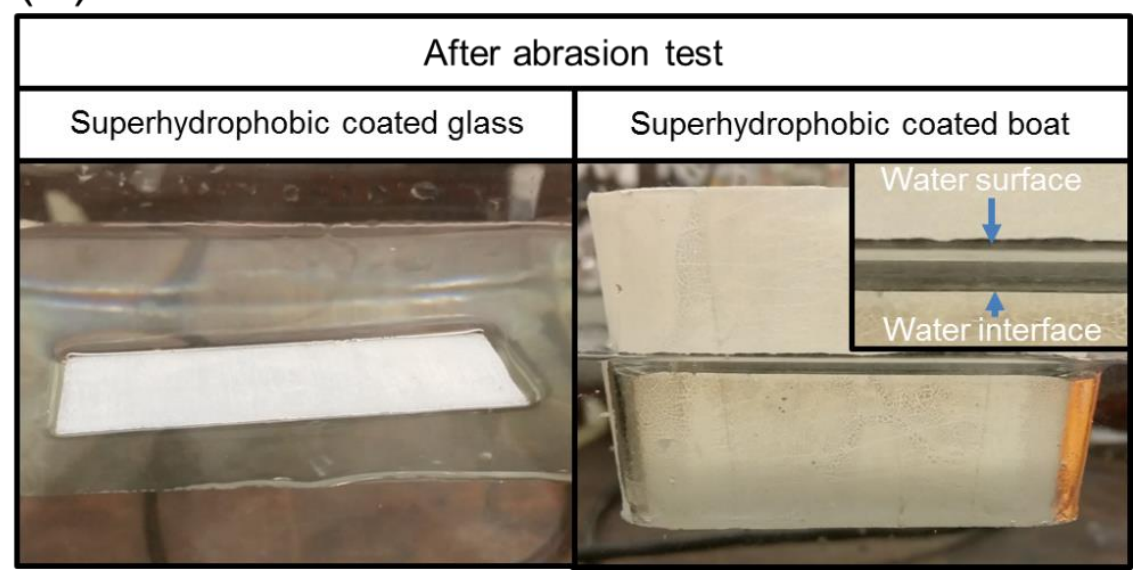

Figure 6. (a) Estimation of water contact angle, contact angle hysteresis, and rolling off angle on treated samples after abrasion test using sand paper, and (b) treated glass sample floating on water and adhesion of water molecule on the surface of the treated boat after $100 \mathrm{~cm}$ abrasion test 


\section{Graphical abstract}

Buoyancy increase and drag-reduction through a simple superhydrophobic coating

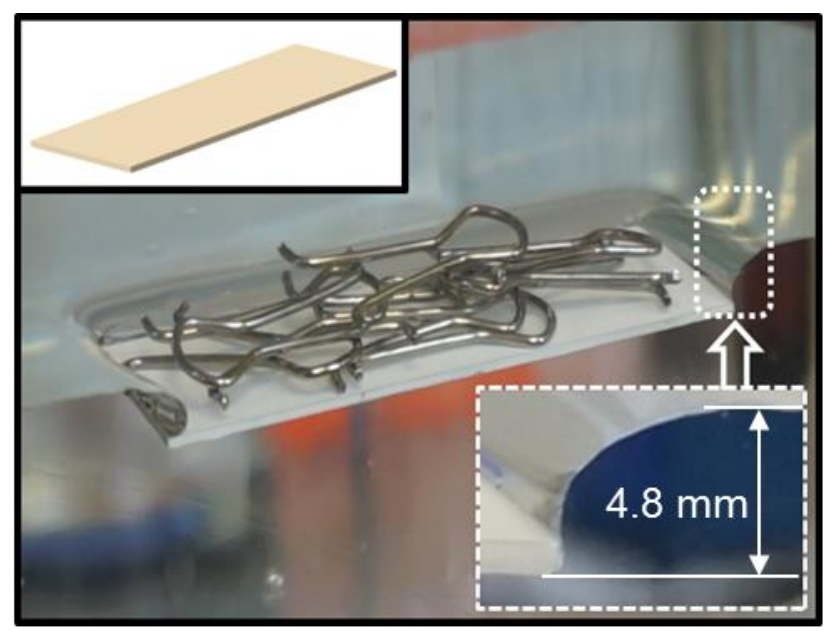

Whole surface coated sample 